\title{
The mechanical properties of human dentin for 3-D finite element modeling: Numerical and analytical evaluation
}

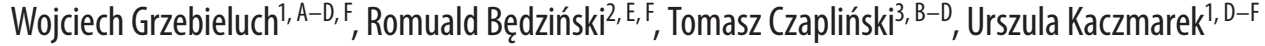 \\ ${ }^{1}$ Department of Conservative Dentistry and Pedodontics, Wroclaw Medical University, Poland \\ ${ }^{2}$ Division of Biomedical Engineering, Department of Mechanical Engineering, University of Zielona Góra, Poland \\ ${ }^{3}$ Nobo Solutions S.A. Wrocław, Poland \\ A - research concept and design; B - collection and/or assembly of data; C - data analysis and interpretation; \\ $D$ - writing the article; $E$ - critical revision of the article; $F$ - final approval of article
}

Address for correspondence

Wojciech Grzebieluch

E-mail:dentysta@poczta.fm

Funding sources

National Science Centre grant N518283740.

Conflict of interest

None declared

Received on August 19, 2016

Revised on September 19, 2016

Accepted on November 30, 2016

DOI

\begin{abstract}
Background. The FEM is often used in investigations of dentin loading conditions; however, its anisotropy is mostly neglected.
\end{abstract}

Objectives. The purpose of the study was to evaluate the anisotropy and the elastic properties of an equivalent homogenous material model of human dentin as well as to compare isotropic and anisotropic dentin FE-models.

Material and methods. Analytical and numerical dentin homogenization according to Luciano and Barbero was performed and E-modulus (E), Poisson's ratios (V) G-modulus (G) were calculated. The E-modulus of the dentin matrix was $28.0 \mathrm{GPa}$, Poisson's ratio (V) was 0.3; finite element models of orthotropic and isotropic dentin were created, loaded and compared using Ansys ${ }^{\circledR} 14.5$ and CodeAster ${ }^{\circledR} 11.2$ software.

Results. Anisotropy of the dentin ranged from 6.9 to 35.2\%. E-modulus and G-modulus were as follows: $\mathrm{E1}=22.0-26.0 \mathrm{GPa}$, E2/E3 = 15.7-23.0 GPa; G12/G13 = 6.96-9.35 GPa and G23 =6.08-8.09 GPa (highest values in the superficial layer). In FEM analysis of the displacement values were higher in the isotropic than in the orthotropic model, reaching up to $16 \%$ by shear load, $37 \%$ by compression and $23 \%$ in the case of shear with bending. Strain values were higher in the isotropic model, up to $35 \%$ for the shear load, $31 \%$ for compression and 35\% in the case of shear with bending. The decrease in the volumetric fraction and diameter of tubules increased the $G$ and $E$ values.

Conclusions. Anisotropy of the dentin applied during FEM analysis decreased the displacements and strain values. The numerical and analytical homogenization of dentin showed similar results.

Key words: finite element method, anisotropy, dentin, homogenization

10.17219/acem/67441

\section{Copyright}

Copyright by Author(s)

This is an article distributed under the terms of the

Creative Commons Attribution Non-Commercial License

(http://creativecommons.org/licenses/by-nc-nd/4.0/) 
Dentin is the main mineralized hard dental tissue supporting structure of the tooth. Its mechanical properties are related to microstructural alterations due to ageing, carious lesions, and restorative procedures.

One of the most common methods of modeling the micromechanical behavior of composites and biological structures is the finite element method (FEM). ${ }^{1-5}$ The FEM enables the modeling of very complex structures (e.g. teeth) and gives an accurate description of the local strain and stress inside the non-homogenous material. ${ }^{3-6}$ Depending on the research topic, finite element (FE) models in macro-, meso- and microscales can be used. ${ }^{3,7-9}$ However, FE models of the biological structure are usually performed using a simplified approach. The employed method may play different roles depending on the model type, physics-related problems or the investigation's objectives. There are a few main mechanical parameters connected with the physical problems and qualities which can have a direct link to the obtained results, including dentin properties, which are usually assumed to be homogeneous, isotropic and linear elastic. ${ }^{3-7}$ Expanding such a method into biomechanics requires a significant and continuous improvement of modeling techniques with respect to the individual anatomic and pathological characteristics of human tissues.

The micromechanical approach enables the prediction of the effective elastic properties of the material, based on its microstructure and the material properties of its components; this process is called homogenization. Numerical homogenization is based on the concept of Representative Volume Element (RVE). RVE is a statistical representation of the microstructure on a macro level. In addition, if the microstructure shows a certain periodicity, as in the case of dentin, the periodic boundary conditions of RVE can be imposed. ${ }^{10}$ One alternative to the numerical method is to use an analytical method in solving the micromechanical problem. Many analytical techniques of homogenization have been based on the equivalent Eigenstrain method, which considers the problem of a single ellipsoidal inclusion embedded in an infinite elastic medium. ${ }^{11}$ Among such analytical methods, one of the most efficient is the Mori-Tanaka method, which takes into account the interactions between inclusions. The main advantage of the Mori-Tanaka method is the delivery of an explicit formula for effective stiffness of the tensor and for local stresses and strains. ${ }^{12,13}$

This approach provides the opportunity to simplify the numerical model and, at the same time, takes into consideration the orthotropic nature of dental tissue. Homogenization enables the prediction of effective elastic properties by considering the mechanical properties of the dentin matrix and dentinal tubules fraction. It can take into account the location-dependent variations of dentinal tubules number and diameter.

Despite many years of development and application of the tooth numerical analysis, anisotropy of both dentin and enamel is neglected. Basically, it can be assumed that the simplified impact on the results of strain distributions remains unknown. It can be regarded reasonable to develop the research methodology reflecting the complex structure and nonlinear properties of dentin during FE analyses and identify how tissue anisotropic properties affect strains and displacement distributions in the complex system of materials with different strain-stress characteristics.

The 2 purposes of this study were to evaluate the degree of anisotropy and determine the qualitative and quantitative elastic properties of a location-dependent homogenous material equivalent to dentin and to calculate the differences between the isotropic and layered orthotropic models of dentin.

\section{Material and methods}

Two experiments were carried out in order to obtain an equivalent homogenous location-dependent material model of human dentin. The whole dentin, due to its composed structure, was divided into 3 layers: top (superficial external $1 / 3$ dentin close to enamel), middle (middle 1/3 dentin) and bottom (internal 1/3 dentin close to dental pulp).

The elastic properties of the entire dentin and its layers were numerically and analytically determined by the homogenization method, and differences between the isotropic and orthotropic models of the dentin tissue were analyzed.

\section{Experiment 1: Dentin homogenization}

\section{Analytical method of dentin homogenization}

The proposed analytical homogenization of the dentin structure was based on the equivalent Eigenstrain method, and took into account the periodic boundary conditions. ${ }^{11}$ The dentin structure was assumed to be a transversely isotropic medium with one axis of symmetry, parallel to the direction of the dentinal tubules. ${ }^{14,15}$ In this case, the micromechanical model used for homogenization was a unidirectional structure with long cylindrical voids embedded in an isotropic matrix. The micromechanical model allowed the strain concentration tensor and corresponding effective stiffness tensor (material properties) of the dentin structure to be determined according to Luciano and Barbero: ${ }^{10}$

$$
\bar{C}=C^{m}-V_{f}\left[\left(C^{m}-C^{f}\right)^{-1}-(-P)\right]^{-1}
$$

Where: $P$ is a 4 rank tensor, which describes the geometry of the voids, microstructures, and the distribution of the voids, $V_{f}$ the volume fraction of the voids (lumen tubules), $C^{f}, C^{m s}$ the 4 rank stiffness tensors of the voids (lumen tubules) and matrix (dentin), both isotropic. 
The input data for human dentin (the dimension and density of the dentinal tubules) used in this study for the analytical homogenization method are listed in Table $1 .{ }^{16-20}$ Young's modulus (E) of the homogenous dentin matrix (intertubular and peritubular dentin) surrounding the dentinal tubules was equal to $28.0 \mathrm{GPa}$ according to Kinney et al. ${ }^{21}$

\section{Numerical method of dentin homogenization}

The prediction of effective material properties of a dentin sample was conducted with the help of the RVE concept, which enabled the modeling of periodic symmetry structures. ${ }^{10}$ For the numerical homogenization procedure the FEM was used to model the RVE of the dentin structure (Fig. 1). The numerical homogenization procedure was performed with the help of the FEM with additional consideration of the periodic boundary condition on the RVE. The periodic boundary conditions were imposed on a representative FE-model of dentin structure, according to Luciano and Barbero, as followed: ${ }^{10}$

$$
u_{i}^{+j}(x, y, z)-u_{i}^{-j}(x, y, z)=\bar{C}_{i k} \Delta x_{k}^{j}
$$

Where: $u_{i}^{+j}, u_{i}^{-j}$ was the imposed displacements of the opposite sides of the RVE cell (side $+j$ and side $-j$ ), $\Delta x_{k}{ }^{j}$ the distance between the opposite sides of the RVE cell, $e_{i k}$ the mean strain in the assessed RVE cell.

This formulation enabled constant distribution of the strain over the whole RVE to be enforced for each direction. At the same time, the strains in other directions were restrained, which enabled the calculation of average stress. This procedure had to be performed 6 times in order to determine all the independent components of the stiffness tensor of the transversely isotropic material. This meant

Fig. 1. Modeling of dentin structure - the method chain from a single RVE cell (dimensions example for tubule diameter $2.4 \mu \mathrm{m}$, axes are visible) (A); dentin layer (B); equivalent orthotropic \& homogenous material model (C); global FE model of the tooth (D)
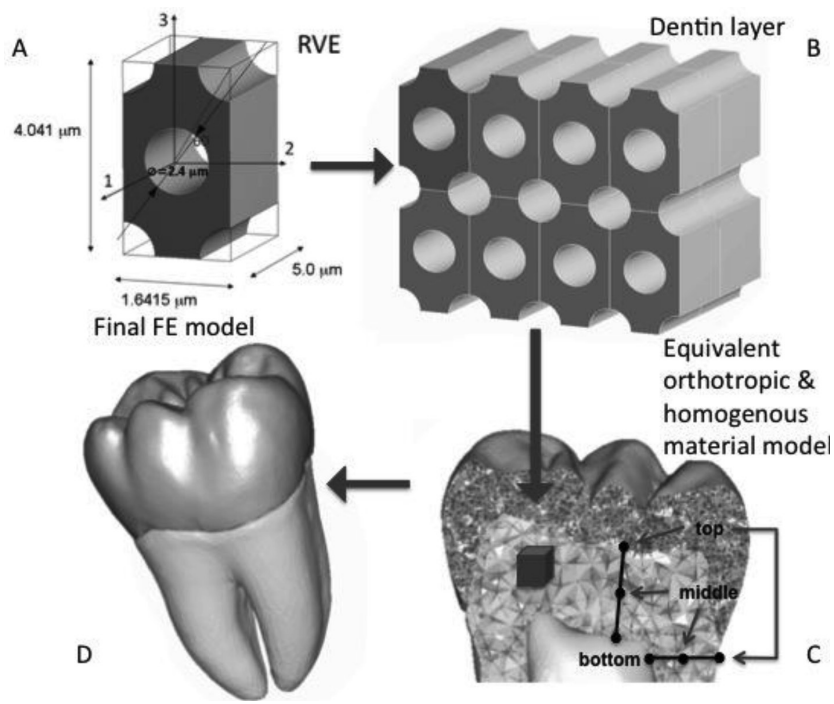

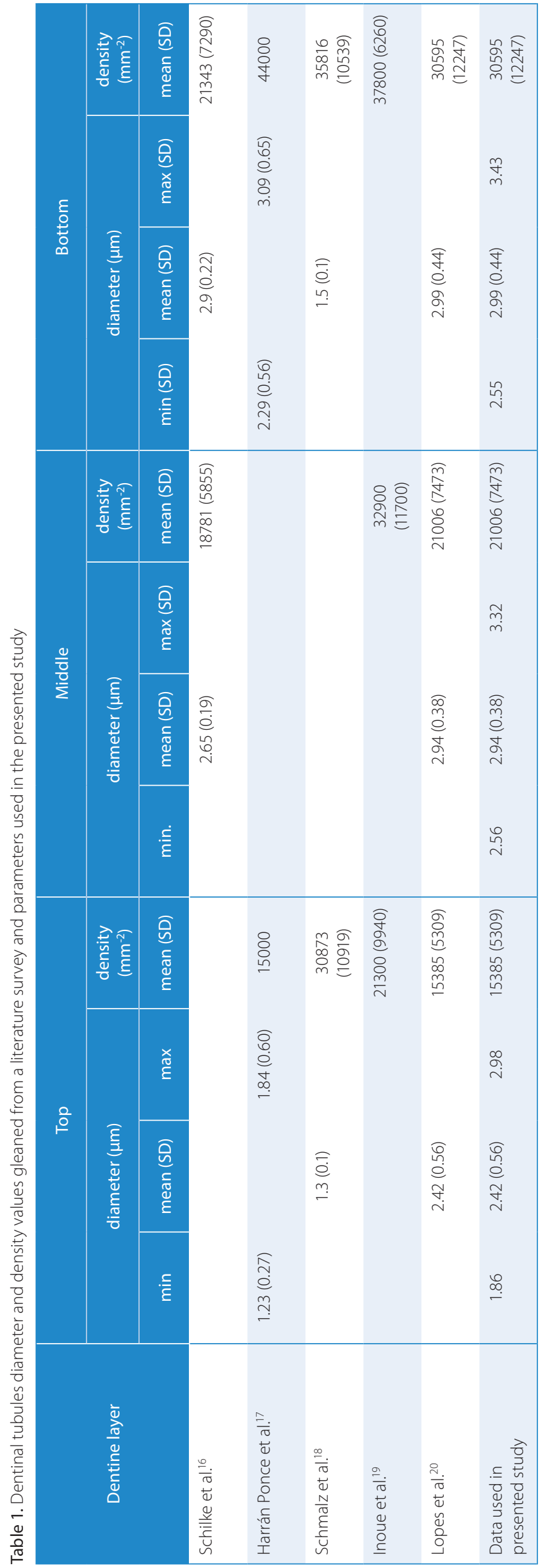


that the 6 independent boundary values problems needed to be solved in order to get a full description of the dentin material. The numerical homogenization procedure was performed with the help of the FEM and Ansys ${ }^{\circledR} 14.5$ (Ansys, Inc., USA) and CodeAster ${ }^{\circledR} 11.2$ (EDF) software. The same input parameters were used for analytical homogenization (Table 1). Young's modulus (E) was equal to 28.0 GPa, according to Kinney et al. ${ }^{21}$ Poisson's ratio was equal to 0.3 .

Three FE-models were prepared: one for each of the top, middle and bottom layers of the dentin structure (Fig. 1). Each model reflected the specific geometry parameters, such as the average diameter of dentin tubules and the volumetric fraction of dentinal tubules in the whole dentin structure.

The results of such analytical and numerical homogenization were used to calculate the anisotropy (\%) of the dentin structure as followed:

$$
a=\left(\frac{E l-E x}{E l}\right) \times 100
$$

Where: $a$ is anisotropy (relative), $E 1$ the reference value of Young's modulus, $E x=E 2=E 3$ value of Young's modulus (perpendicular to E1).

\section{Experiment 2: Finite element analysis of the dentin model}

The 3-dimensional FE models consisting of a simple cube of bulk dentin were created and analyzed using Code Aster 11.4. The cube had $3 \mathrm{~mm}$ sides and was divided into 3 equal parts to represent the top, middle and bottom layers of the dentin structure (Fig. 2). An FE simulation of the evaluated material properties was performed for the orthotropic material model and the

Fig. 2. FE mesh representing the cube model of the dentin; 3 layers of the dentin; axes of the model and loading directions are visible

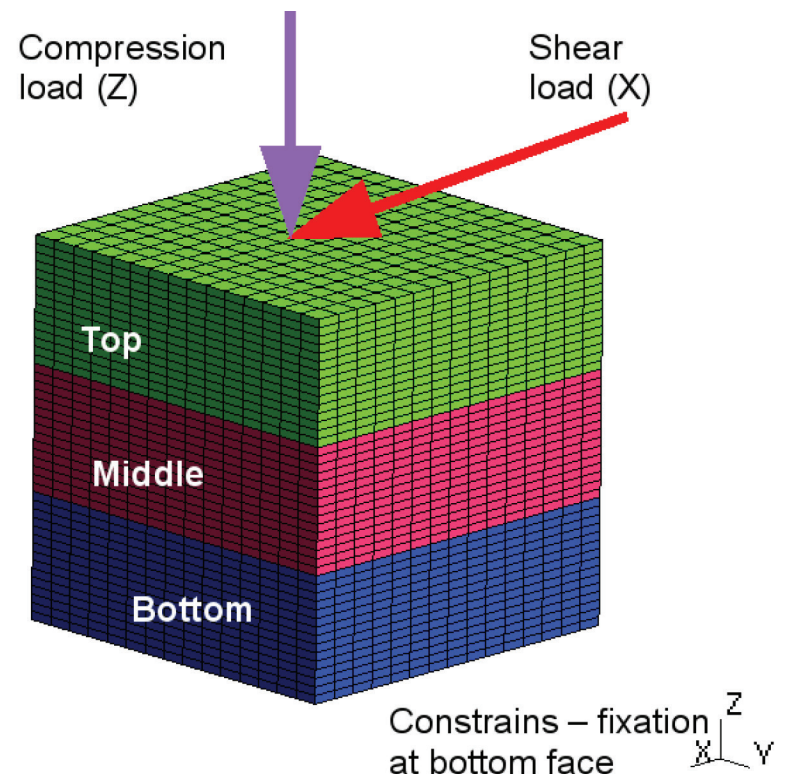

isotropic simplified material model (2 models were analyzed). With both models (iso- and orthotropic), 3 load types were analyzed: (1) a shear only with a shear load of 1.0 MPa applied in X direction on the top surface (the bottom surface nodes were fixed in the X-direction, other nodes were fixed on the $\mathrm{Y}$ and $\mathrm{Z}$ axes), (2) a vertical compression of 1.0 MPa applied towards the $\mathrm{Z}$ axis on the top surface (the bottom surface nodes were fixed in all directions), (3) shear with bending with a shear load of 1.0 MPa applied on the top surface, with bottom surface nodes fixed in all directions and other nodes free in the 3 axes. The input data for human dentin were presented in Table 2. The data for the orthotropic model obtained during experiment 1 was used.

\section{Results}

\section{Experiment 1: Dentin homogenization}

The results of analytical and numerical homogenization were presented in Table 3. An example of the results of the FE analysis of the micromechanical model of the dentin structure was shown in Fig. 3. The effective material properties of the dentin structure were determined for the top, bottom and middle dentin layers (from the pulp up to the dentin's exterior surface). A description of the location of dentin layers was presented in Fig. 1.

\section{Experiment 2: Finite element analysis of the dentin model}

The displacements and strains recorded on the cross section of the model's surface for the isotropic and orthotropic models at different loading conditions were

Fig. 3. Numerical determination of the RVE mean strain - exemplary results of dentin microstructure under: a) and b) tension, c) shear

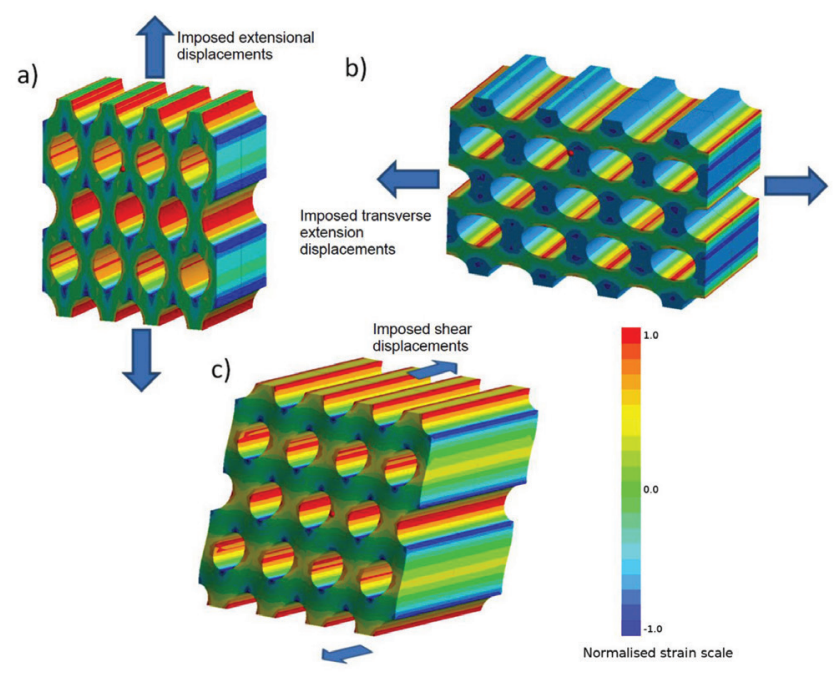


Table 2. Material properties of dentin tissue used during FEM analysis

\begin{tabular}{|c|c|c|c|c|c|c|c|c|c|c|}
\hline \multicolumn{2}{|c|}{ Material designation } & Exx [GPa] & Eyy [GPa] & $\mathrm{Ezz}[\mathrm{GPa}]$ & $\operatorname{vxy}[-]$ & vyz [-] & $\operatorname{vxz}[-]$ & Gxy [GPa] & Gyz [GPa] & Gxz [GPa] \\
\hline \multicolumn{2}{|c|}{ Isotropic dentine } & 18.0 & 18.0 & 18.0 & 0.3 & 0.3 & 0.3 & 6.9 & 6.9 & 6.9 \\
\hline \multirow{3}{*}{$\begin{array}{l}\text { Orthotropic } \\
\text { dentine }\end{array}$} & top & 23.1 & 23.1 & 26.0 & 0.31 & 0.3 & 0.3 & 8.9 & 9.3 & 9.3 \\
\hline & middle & 19.1 & 19.2 & 24.1 & 0.3 & 0.3 & 0.3 & 7.3 & 8.1 & 8.1 \\
\hline & bottom & 15.7 & 15.7 & 22.0 & 0.31 & 0.3 & 0.3 & 6.0 & 7.0 & 7.0 \\
\hline
\end{tabular}

Fig. 4. Finite element analysis of isotropic and orthotropic dentin model: a) displacement during shear loading, b) $1^{\text {st }}$ principal strain during shear loading, c) displacement during vertical compression, d) $1^{\text {st }}$ principal strain during vertical compression, e) displacement during shear with bending,

f) $1^{\text {st }}$ principal strain during shear with bending

$$
\text { a) }
$$

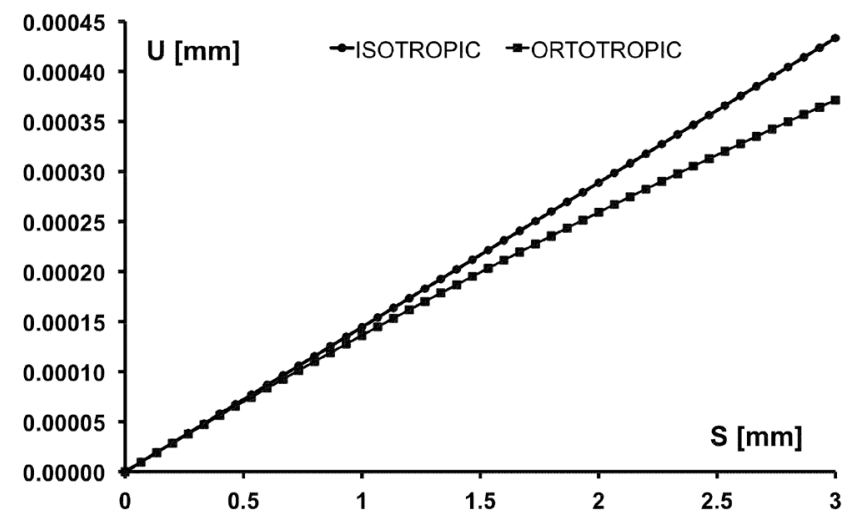

b)

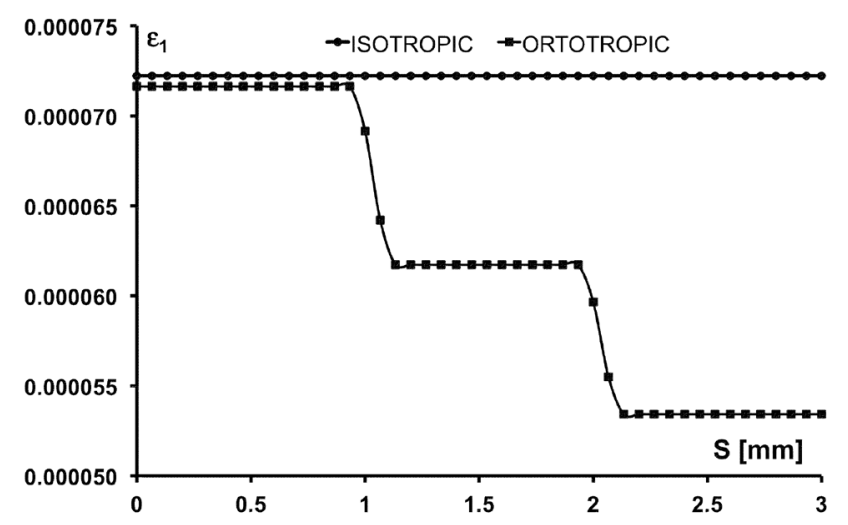

c)

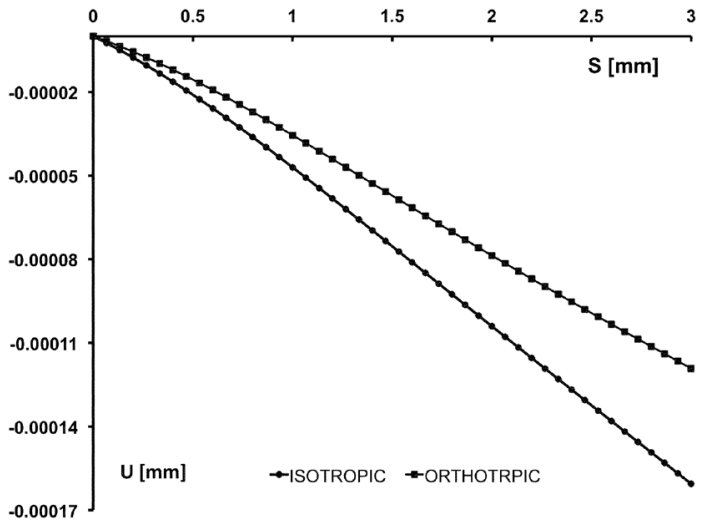

d)

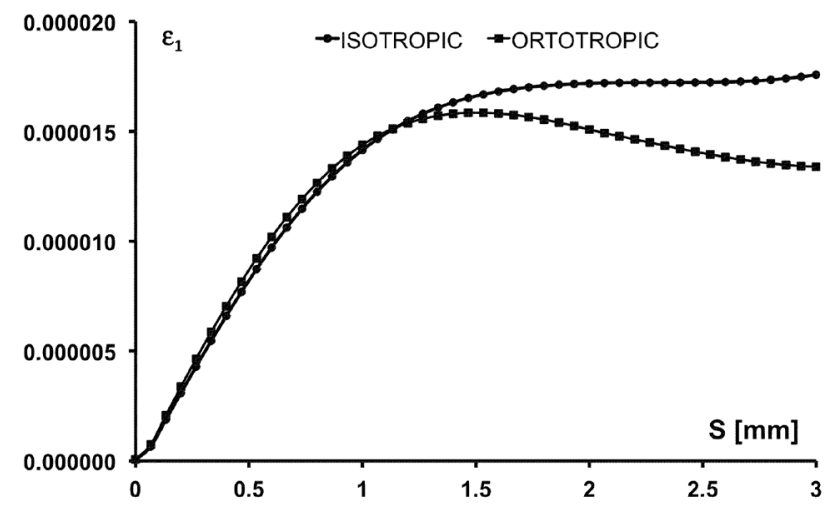

e)

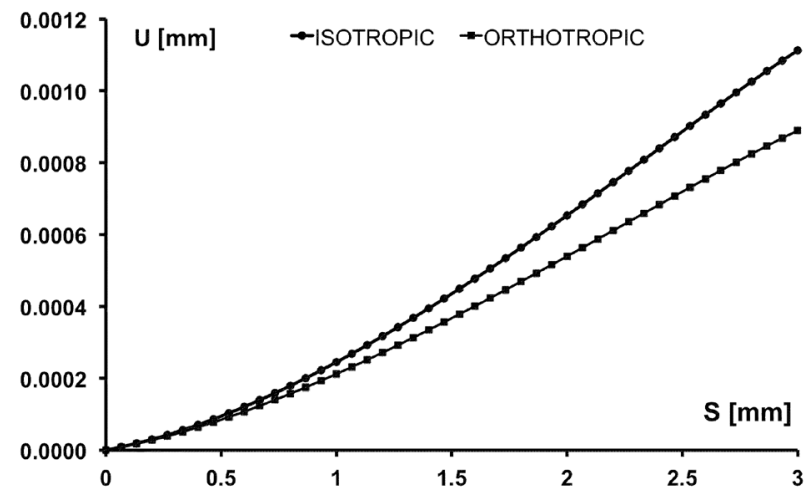

f)

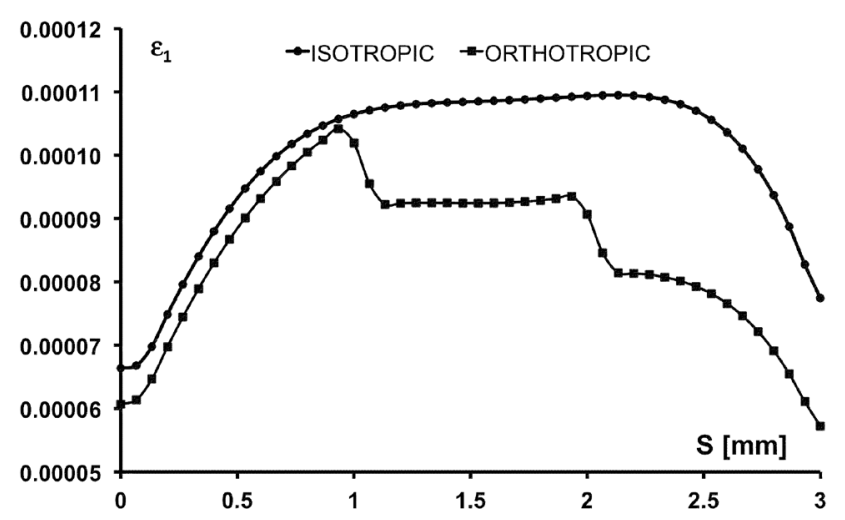


presented in Fig. 4. An analysis of displacements showed higher values in the isotropic model. The differences reached up to $16 \%$ by shear load (Fig. 4a), 37\% by compression (Fig. 4c) and 23\% in the case of shear with bending (Fig. 4e). Similarly, the strain analysis revealed higher strain values in the isotropic model. Strain values were higher, up to $35 \%$ for the shear load (Fig. 4b), 31\% for compression (Fig. 4d) and 35\% in the case of shear with bending (Fig. 4f).

\section{Discussion}

Dentin is a hard tissue that constitutes the bulk of the tooth structure and is composed of approximately $70 \%$ inorganic material (hydroxyapatite crystals), $20 \%$ organic material (mainly collagen type I), and $10 \%$ water by volume. It contains a large number of tubules whose density and diameter decrease from the pulp to the dentinoenamel junction. The dentinal tubules contain the odontoblastic processes, dentinal fluid and nerve fibres, and extend in an S-shaped fashion into the coronal dentin, following a straight course into the radicular dentin. Their wall is formed by very dense, uniformly mineralized peritubular dentin, and they are separated from each other by less densely mineralized intertubular dentin. ${ }^{22}$ The properties of dentin are described as the response of the tooth to the applied loads, and enable the prediction of tooth strain and fracture resistance. The commonly measured effective properties are Young's modulus, tensile and comprehensive strength and fracture toughness, reflecting the complex interactions of the dentin constituents and the microstructure. The properties depend on tubule density and orientation as well as the local density of the mineral phase caused by the remodeling process. The elastic properties of dentin influencing the tooth strength are usually described as stiffness including Young's modulus, shear modulus and Poisson's ratio. ${ }^{14}$

The dentin microstructure, in combination with the whole tooth geometry, causes considerable variations in the results of experimental measurements of the dentin properties. ${ }^{11,14}$ Dental models, like other models from the fields of biology or medicine, have a complex geometry. However, their geometry is relatively easier nowadays thanks to 3D scanners (micro-CT and CT), which offer an easy and fast way to build virtual geometry in any computer-aided engineering (CAE) ${ }^{6,7}$ On the other hand, the physical description of the material and boundary conditions are often significantly simplified. Some typical FE model inputs may be simplified and/or reduced to the essential parts. However, it is relatively difficult to simplify the material properties without changing the natural behavior of the object.

The properties of dentin are usually derived from experimental measurements, which have their own limitations and yield a wide range of results. The complex in- ner structure of the dentin produces anisotropy, which should be taken into account in the studied samples. All difficulties occur especially when the samples are limited because of the shape and size (such as human teeth). Conducting experiments, therefore, involves the use of more sophisticated methods in order to deliver more reliable results. The load and boundary conditions are complex, as are the contact surfaces of the teeth. Therefore, the loads may be represented in non-uniform pressure distribution applied to the suitable sites (physiologically loaded or reflecting in vitro experiments, depending on the modeling type). During FE simulations the most important parameters to set are the mechanical properties. Both quantitative values and qualitative descriptions (e.g. isotropy, anisotropy, axes orientation) are needed. In most of the FEM studies evaluating dental restoration, the dentin is modeled as an isotropic material. The finite element analysis of the dentin model carried out in our study showed higher strain values in the isotropic model. The significant difference observed between the isotropic and anisotropic dentin model revealed the necessity of an estimation of the dentin anisotropy impact on the global FEM model while the tooth restoration analysis.

The homogenization methods are mainly based on the more or less accurate modeling of the non-homogenous composite microstructure and therefore frequently referred to as micromechanical models. Determination of the material parameters (e.g. Young modulus, Poisson's ratio, thermal expansion coefficient) is performed mathematically by averaging the strain tensor of material properties for individual phases over selected volume (RVE).

Micromechanical models can be divided, with regard to the applied methods, into 3 main groups: empirical, analytical and numerical. The analytical models are particularly noteworthy because of the possibility of easy calculations and a small amount of calibration experiments in the empiric models required. Most of the analytical methods of homogenization are based on the equivalent Eigenstrain method, described mathematically as a singular elliptic inclusion in the elastic infinitely large matrix. Similarly, the numerical homogenization determines the effective elastic parameters for non-homogeneous material based on the properties of material phases and their geometry or internal structure. The numerical homogenization also uses the concept of the RVE and assumes that the material is statistically homogeneous at the macro-size level. Numerical micromechanical models are based mainly on the Finite Element Method (FEM). Furthermore, numerical methods of homogenization allow easy inclusion of various geometric details with complicated shapes. Unfortunately, FE modeling of RVE requires significant effort for modeling and CPU computing time.

Our findings showed comparable results for numerical and analytical homogenization and displayed the transversely anisotropic behavior of dentin material. 


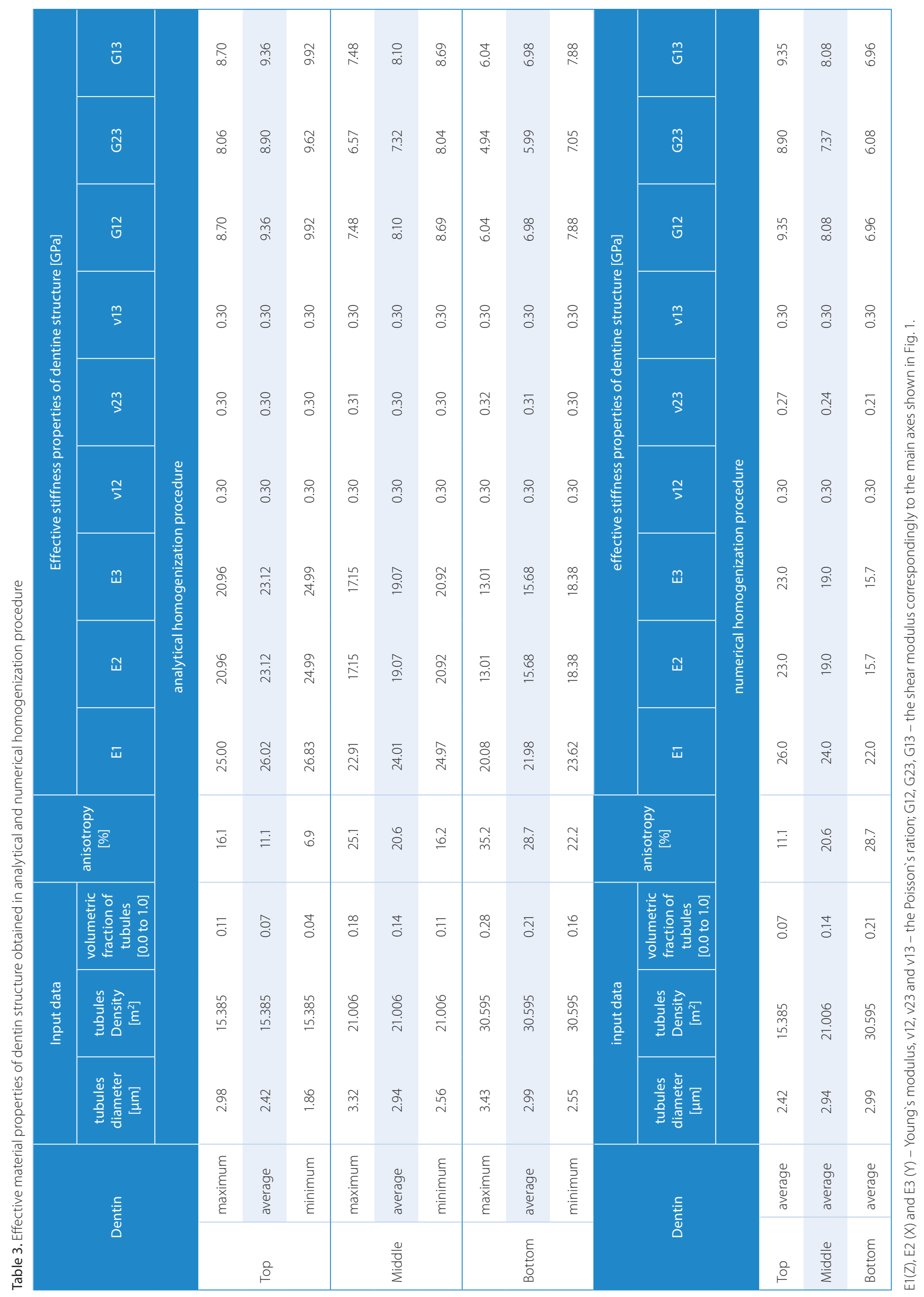


An increase of Young's modulus value (maximum on the top layer) alongside a decrease in the volumetric fraction and diameter of tubules was observed. The increase in E1 of $18 \%$ and E2 and E3 of $47 \%$ on the top layer was also observed. A decrease of the volumetric fraction and diameter of tubules also induced an increase of the shear modulus (G) values. On the top layer of the dentin, the values of G12 and G13 were increased by $34 \%$ and G23 by $48 \%$ (Table 3).

Based on our data we could suggest that the increase of the tubule diameter (and volumetric fraction of tubules) led to the increase in anisotropy and the decrease in the E-modulus value.

Anisotropy of the dentin varied from min. 6.9\% in the top layer to $\max 35.2 \%$ at the bottom layer. The mean values of anisotropy obtained analytically and numerically were comparable but they were higher than relatively low anisotropy ( 10\%) suggested by Kinney et al. ${ }^{23}$ Our results underlined the high impact of the dentinal tubules on the elastic properties and anisotropy of the dentin. This was in opposition to Kinney et al., who stated that the intertubular dentin matrix governed the elastic behavior of dentin, and that the tubules did not introduce elastic anisotropy. ${ }^{21}$ The earlier published dentin tensile strength results confirmed the anisotropic behavior of the dentin and the influence of the tubules direction on the strength. ${ }^{19,24}$

The high anisotropy value may be also explained by the simplification of geometry when dentinal tubules are modeled as straight tubes. However, the influences of the S-shaped dentinal tubules on the results have not been evaluated in this paper. It should also be noted that the tubules' natural orientation could vary widely across a specimen, so potentially an anisotropy may be undetected in a mechanical test of larger specimens. ${ }^{25}$ Miura et al., in order to create multi-scale tooth model, measured $\mathrm{E}$ and $\mathrm{G}$ modules using microindentation. ${ }^{26}$ They obtained high anisotropy values amounting to E 17.07 GPa, G 1.7 GPa longitudinally and E 5.61 GPa and G 6.0 GPa transversally to the tubules. Unfortunately, the information about measuring locations in the tooth structure was not available.

The dentin anisotropy is also detectable during flexural strength tests; Shinno et al. registered the flexural strength of the anti-plane longitudinal vs transverse group that amounted to be $210 \pm 67 \mathrm{MPa}$ and $114 \pm 36$ MPa, respectively. ${ }^{27}$

As mentioned above, the decrease in the volumetric fraction and diameter of the tubules induces the increase of the shear modulus $(G)$ and Young's modulus values. Considering the data obtained with the use of the analytical homogenization procedure, we could state that within the same layer of dentin an increase of the tubules' diameter (i.e. increase of volumetric fraction of tubules) resulted in the increase of anisotropy and the decrease of the E-modulus value. Those results, combined with the large deviation of reported mechanical tests results, suggested the necessity for an analysis of the relation be- tween the tubules' diameter (and volumetric fraction of tubules) and mechanical test data.

Shinno et al. reported that a high density of dentinal tubules also decreased the flexural strength of the dentin. ${ }^{27}$ At the same time, the ageing of the dentin, with the obturation of the tubules, resulting from higher mineralization, could diminish the flexural strength. Panfilov et al. showed higher tensile strength of mature rather than teenage dentin $(549.5 \pm 26.9 \mathrm{MPa}$ vs $445.2 \pm 30.9 \mathrm{MPa}$, respectively). ${ }^{28}$

Within the limitations of this study we can suggest the transversely isotropic behavior of dentin and the anisotropy ranged from 6.9 to $35.2 \%$, depending on the dentin location. The numerical and analytical dentin homogenization presented similar results. The decrease in volume and diameter of tubules resulted in some increase of the shear modulus (G) and Young's modulus (E) values. Both methods give the same level of anisotropy, with relatively small variation. We could assume that the analytical determination of the dentin properties is simpler, more effective and faster, and therefore allows for a recalculation of numerous geometric scenarios. The numerical homogenization requires a new FE model for each new geometric concept leading to a significant increase in the overall cost. However, the analytical model becomes much more complicated (or even unfeasible) for non-parallel or $\mathrm{S}$-shaped tubules, when the numerical homogenization is still applied.

We have attempted to create an anisotropic dentin model imaging its natural nonlinear characteristics and analyze the impact of physiological anisotropy on the dentin strain. The comparison of the isotropic and anisotropic dentin model revealed significant differences in strain, especially in the area with a high density of dentinal tubules. The finite element analysis showed up to over $30 \%$ higher strain values in the isotropic model compared to the anisotropic one. It is worth mentioning that Kishen and Vedantam, as in our study, found that the anisotropy of the dentin and changes in the modules $\mathrm{E}$ and $\mathrm{G}$ (gradients of moduli) during FEM analysis caused significant decrease in displacement and strain values. ${ }^{8}$ Kishen and Vedantam also emphasized the importance of hydrostatic pressure of the dentinal tubule fluid, which was neglected during our study. ${ }^{8}$ The significant difference observed between the isotropic and anisotropic dentin model pointed to the necessity for an estimation of the impact that the dentin anisotropy in the global model has on the FEM analysis results of the tooth restoration.

The anisotropy of the dentin and changes in modules $\mathrm{E}$ and $G$ should be taken into account in future FEM analysis studies. Hence, further study should be directed towards an analysis of the global model of the tooth (whole tooth structure), while applying anisotropy of the dentin and the moduli gradients. This procedure, after model calibration, may be useful in the analysis of the restored teeth and during optimization of restorative process. 


\section{References}

1. Lomov SV, Ivanov DS, Verpoest I, et al. Meso-FE modelling of textile composites: Road map, data flow and algorithms. Compos Sci Technol. 2007;67:1870-1891.

2. Peng $X, C$ ao J. A dual homogenization and finite element approach for material characterization of textile composites. Compos Part B-Eng. 2002;33:45-56.

3. Ferrari M, Sorrentino R, Zarone F, Apicella D, Aversa R, Apicella A. Non-linear viscoelastic finite element analysis of the effect of the length of glass fiber posts on the biomechanical behaviour of directly restored incisors and surrounding alveolar bone. Dent Mater J. 2008;27:485-498.

4. Tajima K, Chen K, Takahashi N, Noda N, Nagamatsu Y, Kakigawa H. Three-dimensional finite element modeling from CT images of tooth and its validation. Dent Mater J. 2009;28:219-226.

5. Yamanel K, Caglar A, Gülsahi K, Ozden UA. Effects of different ceramic and composite materials on stress distribution in inlay and onlay cavities: 3-D finite element analysis. Dent Mater J. 2009;28:661-670.

6. Magne P. Efficient 3D finite element analysis of dental restorative procedures using micro-CT data. Dent Mater. 2007;23:539-548.

7. Poiate IA, Vasconcellosa $A B$, Mori M, Poiate $E$ Jr. 2D and $3 D$ finite element analysis of central incisor generated by computerized tomography. Comput Methods Programs Biomed. 2011;104:292-299.

8. Kishen A, Vedantam S. Hydromechanics in dentin: Role of dentinal tubules and hydrostatic pressure on mechanical stress-strain distribution. Dent Mater. 2007;23:1296-1306.

9. Miuraa J, Maedaa Y, Nakaib H, Zakob M. Multiscale analysis of stress distribution in teeth under applied forces. Dent Mater. 2009; 25:67-73.

10. Luciano R, Barbero EJ. Formulas for the stiffness of composites with periodic microstructure. Int J Solids Structures. 1994;31:2933-2944.

11. Eshelby JD. The determination of the elastic field of an ellipsoidal inclusion and related problems. Proc $R$ Soc A. 1957;241:376-396.

12. Mori T, Tanaka K. Average stress in matrix and average elastic energy of materials with misfitting inclusions. Actas Metall. 1973;21:571-574.

13. Gommers B, Verpoest I, Van Houtte P. The Mori-Tanaka method applied to textile composite materials. Acta Mater. 1998;46:2223-2235.

14. Kinney J H, Marshall SJ, Marshall GW. The mechanical properties of human dentin: A critical review and re-evaluation of the dental literature. Crit Rev Oral Biol Med. 2003;14:13-29.

15. Inoue T, Takahashi $H$, Nishimura F. Anisotropy of tensile strengths of bovine Dentin regarding dentinal tubule orientation and location. Dent Mater J. 2002;21:32-42.
16. Schilke R, Lisson JA, Bauss O, Geurtsen W. Comparison of the number and diameter of dentinal tubules in human and bovine dentin by scanning electron microscopic investigation. Arch Oral Biol. 2000;45:355-361.

17. Harrán Ponce E, Canalda Sahli C, Vilar Fernandez JA. Study of dentinal tubule architecture of permanent upper premolars: Evaluation by SEM. Aust Endod J. 2001;27:66-72.

18. Schmalz G, Hiller KA, Loys J. Nunez LJ, Stoll J, Weis K. Permeability characteristics of bovine and human dentin under different pretreatment conditions. J Endod. 2001;27:23-30.

19. Inoue S, Pereira PN, Kawamoto C, et al. Effect of depth and tubule direction on ultimate tensile strength of human coronal dentin. Dent Mater J. 2003;22:39-47.

20. Lopes MB, Sinhoreti MA, Gonini Júnior A, Consani S, McCabe JF. Comparative study of tubular diameter and quantity for human and bovine dentin at different depths. Braz Dent J. 2009; 20:279-283.

21. Kinney JH, Balooch M, Marshall GW, Marshall SJ. A micromechanics model of the elastic properties of human dentin. Arch Oral Biol. 1999;44:813-822.

22. Schroeder HE. Oral structural biology: Embryology, structure, and function of normal hard and soft tissues of the oral cavity and temporomandibular joints. Thieme Medical Publishers, Inc., New York. 1991:103-118.

23. Kinney JH, Gladden JR, Marshall GW, Marshall SJ, So JH, Maynard JD. Resonant ultrasound spectroscopy measurements of the elastic constants of human dentin. J Biomech. 2004;37:437-441.

24. Inoue T, Takahashi H, Nishimura F. Anisotropy of tensile strengths of bovine dentin regarding dentinal tubule orientation and location. Dent Mat J. 2002;21:32-43.

25. Chowdhary N, Subba Reddy VV. Dentin comparison in primary and permanent molars under transmitted and polarised light microscopy: An in vitro study. J Indian Soc Pedod Prev Dent. 2010; 28:162-172.

26. Miur J, Maeda Y, Nakai H, Zako M. Multiscale analysis of stress distribution in teeth under applied forces. Dent Mater. 2009;25:67-73.

27. Shinno $Y$, Ishimoto $T$, Saito $M$, et al. Comprehensive analyses of how tubule occlusion and advanced glycation end-products diminish strength of aged dentin. Sci Rep. 2016;6:19849, doi: 10.1038/ srep19849.

28. Panfilov P, Zaytsev D, Antonova OV, Alpatova V, Kiselnikova LP. The difference of structural state and deformation behavior between teenage and mature human dentin. Int J Biomater. 2016;2016:6073051. doi: 10.1155/2016/6073051. Epub 2016 Feb 16. 